Conclusions QT/RR slope of DCM sudden death group was higher than DCM non sudden death and Con group significantly. OT/RR slope show high predicting value for sudden death in DCM patients independently or combined with NSVT or LVEF.

\section{e0336 EFFECT OF TAURINE ON VASCULAR SMOOTH MUSCLE CELLS APOPTOSIS IN ATHEROSCLEROTIC RABBITS}

doi:10.1136/hrt.2010.208967.336

${ }^{1}$ Zhai Gui-Lan, ${ }^{2}$ Zhu Huan-Lin, 'Dong Zhi-Hua. ${ }^{1}$ The First Affiliated Hospital of Liaoning Medical college; ${ }^{2}$ The 205 Hospital of Chinense PLA, Liaoning

Objective To study the effect of taurine on the apoptosis of vascular smooth muscle cells in atherosclerotic model of rabbits and the mechanism of anti-atherosclerosis.

Methods 21 male Japanese white rabbits were divided into three groups: normol control group, high cholesterol group and taurine group. The normal control group were fed with standard chow diet and two other groups with a high fat diet. The taurine group were fed with taurine solution once a day, two other groups were given normal saline gastric feeding. Twelve weeks later the modelling was determined successful, all rabbits were killed with air embolism method and exposed the heart, isolated and cut aorta from aortic valve to the bifurcation of abdominal aorta blood vessel. Observing the pathomorphological changes in aorta wall and ultra-structures of VSMCS were observed by electronic microscopy, the apototic rate of VSMCs detected by flow cytometry, expression of bcl-2 and bax proteins were detected by immunohistochemistry and expression of caspase- 3 proteins were detected by Western blot.

Results The aortic intima of normal control group was smooth, no plaque formation; the high cholesterol group was uneven and rough, there were many needle-like white mastoid processes, some fused into pieces; the above-mentioned diseases of the taurine group were less. Three-tier structure of the normal control group were observed clearly through light microscope $(\mathrm{HE} \times 400)$, vessel wall was smooth and VSMCs arranged regularly; the intima of the high cholesterol group was thicker significantly and irregular foam cells were aggregation, a large number of lipid could be seen at elastic plates and cell gap, smooth muscle cells arranged irregularly; threetier structure of the vessel wall in the taurine group could be seen clearly, the intima is thick partly, foam cells were less, lipid is deposition rarely, smooth muscle cells are still arranged neatly, the intima and intima-to-media were significantly decrease $(p<0.01)$. In high cholesterol group the apoptotic rate of VSMCs was higher than that in normal control group $(p<0.01)$, the expression of bcl-2 proteins was lower $(p<0.01)$, but the expression of bax and caspase3 proteins was higher $(\mathrm{p}<0.01)$. The visible atheromatous plaque which caused the serious stenosis were observed and the apoptotic VSMCs were more in the atheromatous plaque in high cholesterol group. In taurine group the apoptotic rate of VSMCs was lower than that in high cholesterol group $(p<0.01)$, the expression of bcl-2 proteins was higher $(p<0.01)$, but the expression of bax and caspase3 proteins were lower $(p<0.01)$. The atheromatous plaque were decreased and the stenosis were reduced, and the apoptotic VSMCs were less and not typical.

Conclusions Taurine can prevent the formation of atherosclerosis and inhibit the apoptosis of VSMCs in the atherosclerotic plaque by regulating the bcl-2, bax and caspase- 3 proteins.

\section{e0337 MALLEOLUS ARM INDEX CLINICAL PRACTICE ANALYSIS}

\section{doi:10.1136/hrt.2010.208967.337}

Liping Yang, Songsheng Yang, Zongning Chen, Gutian Zhao, Yin Wei, Yuan Zhao, Xuehua Zhao. Lijiang Municipal People's Hospital

Objective To evaluate $\mathrm{ABI}$ abnormalities and associated risk fators, we measured ankle brachial index (ABI) of cardiovascular- event high-risk patients. The first step enquiries the cardiovascular-event high-risk to win high limit of the ABI abnormality.

Methods We measured the ABI of 773 cases of hospitalised cardiovascular-event high-risk patients. $\mathrm{ABI}<0.9$ and $\mathrm{ABI}>1.3$ are defined as abnormal. Collected data and process statistics analysis to investigate the independent risk factors of $\mathrm{ABI}$.

Results The incidence of abnormal $\mathrm{ABI}$ is $54 \%$. Among them $\mathrm{ABI}<0.9$ have $12.4 \%$, Independent risk factors to predict $\mathrm{ABI}$ abnormalities are: sex, age, diabetes, creatinine abnormalities, mellitus, hypertension, high cholesterol, high smoking. In cardiovascular-event high-risk $A B I<0.9$ and $A B I>1.3$ all show obvious difference. TheABI no show obvious difference in difference clan.

Conclusions In patients with cardiovascular-event high-risk $\mathrm{ABI}<0.9$ and $\mathrm{ABI}>1.3$ are abnormal. $\mathrm{ABI}$ abnormalities are particularly prevalent in the old, smokers and patients with hypertension, diabetes or dyslipidemia. The difference of ABI between each clan did not show statistics to learn the difference.

\section{e0338 ABLATION OF LEFT-VARIATED DUAL ATRIOVENTRICULAR NODAL PATHWAY IN CORONARY SINUS}

doi:10.1136/hrt.2010.208967.338

${ }^{1}$ Huang Weibin, ${ }^{2}$ Hong Jiang, ${ }^{1}$ Wang Yan, 'Zhou Faguang, 'Zeng Zhaopin, ${ }^{3}$ Wang Lexin, ${ }^{2}$ Sun Baogui. ${ }^{1}$ Xiamen University Zhongshan Hospital; ${ }^{2}$ Shanghai Jiaotong University Affiliated First Peoples Hospital; ${ }^{3}$ School of Biomedical Sciences Charles Sturt University

Atrioventricular nodal reentrant tachycardia (AVNRT) is the most common paroxysmal supraventricular tachycardia. It is well accepted that the mechanism of AVNRT is reentry associated with dual or multiple atrioventricular nodal (AVN) pathway. Typical AVNRT pathways including fast and slow pathways are confined in right atrium. Radiofrequency catheter ablation slow pathway, occasionally fast pathway, has become the definitive treatment of choice for most symptomatic patients. Besides typical AVNRT, there exists some atypical AVNRT with various manifestations. Several groups have reported successful ablation of leftward dual AVN pathway in the left side of the heart. We present one case of leftvariated AVN as well as dual AVN pathway. Routine ablation methods failed to eliminate the tachycardial. Detailed electrical physiological study showed that His Bundle potential was minimal recorded in the right atrium septum but was prominent when recorded in the left septum using a catheter via transaortic approach. Left variated dual AVN pathway was considered. Since routine methods were unsuccessful, an ablating was forward deep into coronary sinus when a target with $\mathrm{A} / \mathrm{V} \approx 1 / 4$ and without His bundle electrogram was mapped. A small energy attempt showed effective and the tachycardia was successfully eliminated by ablation of slow pathway deep in the coronary sinus.

\section{E0339 ANTERIOR SPINAL ARTERY SYNDROME DUE TO CARDIAC TAMPONADE AFTER PERCUTANEOUS CORONARY INTERVENTION-A CASE REPORT}

doi:10.1136/hrt.2010.208967.339

${ }^{1}$ Tong Hong, ${ }^{2}$ Dan Qiuhong, ${ }^{2}$ Chen Lei. ${ }^{1}$ Taizhou First People Hospital; ${ }^{2}$ Taizhou Center Hospital

Objective Delayed cardiac tamponade is an uncommon complication of percutaneous coronary intervention (PCI). Anterior spinal artery syndrome (ASAS) induced by cardiac arrest due to cardiac tamponade is rare. We report such a case and discussed the causes and prevention measures.

Case report This is a 78 year-old man admitted for exertion angina for 3 months. He had implanted with a VVI cardiac pacer 3 months 